\title{
Title: Photoperiodic control of seasonal growth is mediated by ABA acting on cell-cell communication
}

Authors: S. Tylewicz ${ }^{1, \dagger}$, A. Petterle ${ }^{1}$, S. Marttila ${ }^{2}$, P. Miskolczi ${ }^{1}$, A. Azeez ${ }^{1,3}$, R. K. Singh ${ }^{1}$, J.

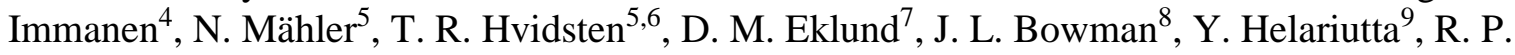

Bhalerao $^{1, *}$

\author{
Affiliations: \\ ${ }^{1}$ Umeå Plant Science Centre, Department of Forest Genetics and Plant Physiology, Swedish \\ University of Agricultural Sciences, SE-901 87 Umeå, Sweden \\ ${ }^{2}$ Department of Plant Protection Biology, Swedish University of Agricultural Sciences, Box 102, \\ ${ }^{3}$ Plant Molecular Biology Lab, Jain R\&D Lab, Agri Park, Jain Hills, Shirsoli Road, Jalgaon, \\ India \\ ${ }^{4}$ Department of Biosciences, Institute of Biotechnology, University of Helsinki, Viikinkaari 1 \\ (P.O.Box 65), Helsinki, Finland \\ 15 Umea Plant Science Centre, Department of Plant Physiology, Umeå University, SE-901 87, \\ Umeå, Sweden \\ ${ }^{6}$ Faculty of Chemistry, Biotechnology and Food Science, Norwegian University of Life Sciences, \\ Ås, Norway \\ ${ }^{7}$ Department of Plant Ecology and Evolution, Evolutionary Biology Centre, Uppsala University, \\ ${ }^{8}$ School of Biological Sciences, Monash University, Melbourne, VIC, Australia \\ ${ }^{9}$ Sainsbury Laboratory, Cambridge University, Bateman Street, Cambridge, United Kingdom \\ *Corresponding author. Email: rishi.bhalerao@slu.se \\ $\dagger$ Present address: Department of Plant and Microbial Biology, University of Zürich, \\ 25 Zollikerstrasse 107, 8008 Zürich, Switzerland
} 10 SE-230 53 Alnarp, Sweden 20 SE-75236 Uppsala, Sweden

\footnotetext{
Abstract: In temperate and boreal ecosystems, seasonal cycles of growth and dormancy allow perennial plants to adapt to winter conditions. We show, in hybrid aspen trees, that photoperiodic regulation of dormancy is mechanistically distinct from autumnal growth cessation. Dormancy sets in when symplastic inter-cellular communication through plasmodesmata is blocked by a process dependent upon the phytohormone abscisic acid (ABA). The communication blockage prevents growth-promoting signals from accessing the meristem. Thus, precocious growth is
} 
disallowed during dormancy. The dormant period, which supports robust survival of the aspen tree in winter, is due to loss of access to growth promoting signals.

One Sentence Summary: Photoperiodic signals transduced via plant hormone abscisic acid enables winter survival by dormancy establishment in trees

Main Text:

Dormancy protects meristematic cells of perennial plants in temperate and boreal ecosystems by preventing growth during winter. Release from dormancy enables reinitiation of growth when favorable conditions return in spring (1). Shorter photoperiods as winter approaches (2) induce growth cessation, formation of a bud enclosing the arrested leaf primordia and shoot apical meristem (SAM) (Fig. 1A), and bud dormancy (3, 4). Longer photoperiods alone cannot promote growth in dormant buds; prolonged exposure to low temperatures is required to release dormancy $(5,6)$. We show that blockage of symplastic communication mediated by the action of abscisic acid (ABA) is part of the photoperiodically controlled dormancy mechanism in hybrid aspen. aspen buds $(4,7)$. ABA regulates dormancy (8). Therefore, we probed ABA's role in photoperiodic control of bud dormancy. First, we generated hybrid aspen plants with reduced ABA responses by expressing the dominant negative abil-1 allele of $A B I 1$, a key ABA-signaling gene (9). Those hybrid aspens that expressed abil-1 had reduced ABA responses, manifested by weak induction of the ABA-inducible gene KIN2, compared to wild-type (WT) controls (Fig. S1). We then assessed bud dormancy by exposing WT and abil-1 plants to 11 weeks of short photoperiod followed by transfer to long photoperiod without low temperature treatment required for dormancy release. Both WT and abil-1 plants ceased growth and set buds after 4 
weeks of short photoperiod (Fig 1A-C), but after 11 weeks of short photoperiod followed by long photoperiod, WT buds remained dormant, whereas abil-1 buds reactivated growth within 11-15 days (Fig. 1D-F). Thus, attenuation of ABA responses compromised photoperiodic control of bud dormancy and not growth cessation.

We investigated transcriptomic responses to short photoperiod in WT and abil-1 apices to understand ABA mediated control of dormancy. After 6 and 10 weeks of short photoperiod, respectively, we detected: 9290 and 3053 differentially expressed genes in WT and 10514 and 2149 in abil-1 (line 1) apices (Table S1). A large number of transcripts for plasmodesmata associated proteins responded to short photoperiod. Plasmodesmata closure (by callosic dormancy sphincters) correlates with dormancy and their opening with dormancy release in diverse plants, including hybrid aspen and charophycean algae such as Chara $(6,10,11)$. Of 187 poplar homologs of Arabidopsis genes encoding proteins enriched in plasmodesmata (12), 62 and 47 were induced after 6 and 10 weeks in WT apices, respectively, and of these 53.2 and $76.6 \%$ were differentially expressed in abil-1 relative to WT apices at these time-points (Table S2). Expression of GERMIN-LIKE 10, REMORIN-LIKE 1 and 2, implicated in plasmodesmata function (13), and CALLOSE SYNTHASE 1, required for callose deposition (6), was progressively upregulated, while that of GH17-39, a glucanase implicated in sphincter removal (6), was downregulated in WT apices after 6 and 10 weeks of short photoperiod. These genes showed altered response to short photoperiod in abil-1 plants (Fig. S2). Thus, ABA mediates short photoperiod response of plasmodesmata-related transcriptome.

Transcriptomic analysis prompted us to investigate ABAs' role in plasmodesmata closure (Fig 1 G-O). Under long photoperiod, WT and abil-1 lines 1 and 3 had similar frequencies of 'closed' plasmodesmata with dormancy sphincters (12.5 versus 17.4 and $13.5 \%$, respectively). After 5 
weeks of short photoperiod, corresponding frequencies were $78 \%$ in WT and 5.5 and $17.4 \%$ in abil-1 apices, respectively, and after 10 weeks frequencies increased to $83.6 \%$ in WT plants, but fell to 2.2 and $0.5 \%$ in abil-1 lines 1 and 3, respectively. Thus, ABA mediates plasmodesmata closure in response to short photoperiod. Plasmodesmata closure is not required for growth cessation (as growth cessation occurs in abil-1 plants) and indicates association of plasmodesmata closure with bud dormancy, both being mediated by the same factor, ABA. To investigate ABA-mediated plasmodesmata closure's role in short photoperiod-induced dormancy, we over-expressed PDLP1, which impairs trafficking via plasmodesmata (14) and phenocopying plasmodesmata blockage by dormancy sphincters, in abil-1 plants (Fig. S3). Both abil-1/PDLP1 double transformants and parental abil-1 plants ceased growth and formed buds under short photoperiod (Fig. 2A-C), but subsequent exposure to long photoperiod only reactivated growth in the latter (Fig. 2D-F). Thus, PDLP1 expression suppressed abil-1 plants' bud dormancy phenotype, although, KIN2 expression responses to ABA remained attenuated in abi1-1/PDLP1 (Fig. S4). Thus, expression of PDLP1 was sufficient to restore bud dormancy in abil-1/PDLP1 plants without the restoration of general ABA responses.

PICKLE (PKL) is an antagonist of polycomb repression complex 2, implicated in seed dormancy $(15,16) . P K L$ expression was downregulated in WT plants but upregulated in abil-1 plants under short photoperiod (Fig. S5). Hence we investigated whether $P K L$ could be involved in plasmodesmata closure and dormancy regulation mediated by ABA. Thus, we examined plasmodesmata in abil-1 plants with suppressed $P K L$ activity (abil-1/PKLRNAi) (Fig. S6). Under long photoperiod, frequencies of plasmodesmata with dormancy sphincters were comparable in abil-1 (13.1\%) and abil-1/PKLRNAi lines 9 (19.4\%) and 11 (18.4\%) (Fig. 3AC). After 5 weeks of short photoperiod, the frequencies increased in the abil-1/PKLRNAi lines 
(to $34.4 \%$ and $28.5 \%$, respectively), but not abil-1 plants (16.4\%) (Fig. 3D-F). After 10 weeks of short photoperiod, the frequencies further increased in abil-1/PKLRNAi lines 9 and 11 to $84.6 \%$ and $74.5 \%$, respectively, but fell in abil-1 plants (5.2\%) (Fig. 3G-I). PKL downregulation in abil-1/PKLRNAi also suppressed expression defects of plasmodesmata markers evident in abil1 plants (Fig. S7). While both abil-1 and abil-1/PKLRNAi plants ceased growth and set buds (Fig. 3J-L), abi1-1/PKLRNAi buds remained dormant and did not reactivate growth (unlike nondormant abil-1 buds) after long photoperiod exposure following 11 weeks of short photoperiod (Fig. 3M-O). Thus, PKL downregulation restores plasmodesmata closure and bud dormancy defects in abil-1 plants, suggesting that ABA mediates plasmodesmata closure and bud dormancy by suppressing $P K L$.

Plasmodesmata closure could mediate dormancy by limiting access of SAM to growth promotive signals. We investigated this hypothesis by analyzing responses of WT and abil-1 buds to FLOWERING LOCUS T 1 (FT1), a seasonal growth regulator induced during dormancy release and before bud growth resumes $(6,17)$. We grafted scions of WT and abil-1 plants exposed to 10 weeks of short photoperiod (to induce plasmodesmata closure and dormancy) onto rootstocks of FT1-expressing plants (18). Although buds of WT scions did not reactivate growth, new leaves emerged from buds of abil-1 scions under continued short photoperiod (Fig. 4). Thus, plasmodesmata closure, as in WT plants, was associated with buds' failure to respond to FT1 or FT1-derived growth promotive signals, corroborating the involvement of plasmodesmata in photoperiodic control of ABA-mediated bud dormancy.

Thus short photoperiods suppressed FT, which causes growth cessation, and amplifies the ABA response by enhancing levels of $\mathrm{ABA}$ and $\mathrm{ABA}$ receptors $(4,7)$. $\mathrm{ABA}$ suppresses $\mathrm{PKL}$ and induces callose synthase to block plasmodesmata and maintains these blockages by repressing 
antagonistic glucanases (Fig. S8). Hence, attenuating ABA responses not only results in a failure to induce plasmodesmata closure at dormancy onset, but also in fewer subsequently closed plasmodesmata. Plasmodesmata closure by $P K L$ downregulation or $P D L P 1$ expression, which both target cell-cell communication, suppresses dormancy defects in abil-1 plants. PDLP1 expression restores dormancy without suppressing ABA response defects in abil-1 plants. Thus, plasmodesmata closure is essential to dormancy and occurs downstream of ABA-mediated control of dormancy in response to shorter photoperiods.

With plasmodesmata closed, growth arrest is maintained even in the presence of growth promoting signals. Re-opening of closed plasmodesmata in dormant buds occurs slowly and only after prolonged exposure to low temperature. Hence, dormancy prevents precocious activation of growth. On the other hand, in the absence of dormancy and plasmodesmatal closure, growth cessation induced by short photoperiod can be quickly reversed. Thus, dormancy, unlike growth cessation, adds robustness to the mechanism that is crucial for perennial survival and longevity in the face of changing seasons.

\section{References and Notes:}

1. R. K. Singh, T. Svystun, B. AlDahmash, A. M. Jönsson, R. P. Bhalerao, Photoperiod- and temperature-mediated control of phenology in trees - a molecular perspective. New Phytol. 213, 511-524 (2017).

2. C. J. Weiser, Cold resistance and injury in woody plants. Science 169, 1269-1278 (1970).

3. J. E. Olsen et al., Ectopic expression of oat phytochrome A in hybrid aspen changes critical daylength for growth and prevents cold acclimatization. Plant J. 12, 1339-1350 (1997). 
4. T. Ruttink et al., A molecular timetable for apical bud formation and dormancy induction in poplar. Plant Cell 19, 2370-2390 (2007).

5. A. Espinosa-Ruiz et al., Differential stage-specific regulation of cyclin-dependent kinases during cambial dormancy in hybrid aspen. Plant J. 38, 603-615 (2004).

6. P. L. Rinne et al., Chilling of dormant buds hyperinduces FLOWERING LOCUS T and recruits GA-inducible 1,3-beta-glucanases to reopen signal conduits and release dormancy in Populus. Plant Cell 23, 130-146 (2011).

7. A. Karlberg et al., Analysis of global changes in gene expression during activity-dormancy cycle in hybrid aspen apex. Plant Biotechnology 27, 1-16 (2010).

8. S. Penfield, J. King, Towards a systems biology approach to understanding seed dormancy and germination. Proceedings of the Royal Society B: Biological Sciences 276, 3561-3569 (2009).

9. J. Leung et al., Arabidopsis ABA response gene ABI1: features of a calcium-modulated protein phosphatase. Science 264, 1448-1452 (1994).

10. V. A. Shepherd, P. B. Goodwin, Seasonal patterns of cell-to-cell communication in Chara corallina Klein ex Willd. I. Cell-to-cell communication in vegetative lateral branches during winter and spring. Plant Cell Environ. 15, 137-150 (1992).

11. L. C. Jian, L. H. Sun, Blocking and breaking of the plasmodesmata in winter wheat seedlings in midwinter period and their role for stabilizing cold resistant ability. Botany Research 6, 157-162 (1992).

12. L. Fernandez-Calvino et al., Arabidopsis plasmodesmal proteome. PLoS One 6, e18880 (2011). 
13. S. Raffaele, E. Bayer, S. Mongrand, Upregulation of the plant protein remorin correlates with dehiscence and cell maturation: a link with the maturation of plasmodesmata? Plant Signal Behav 4, 915-919 (2009).

14. C. L. Thomas, E. M. Bayer, C. Ritzenthaler, L. Fernandez-Calvino, A. J. Maule, Specific targeting of a plasmodesmal protein affecting cell-to-cell communication. PLoS Biology 6, 01800190 (2008).

15. E. Aichinger et al., CHD3 proteins and polycomb group proteins antagonistically determine cell identity in Arabidopsis. PLoS Genet. 5, e1000605 (2009).

16. D. Bouyer et al., Polycomb repressive complex 2 controls the embryo-to-seedling phase transition. PLoS Genet. 7, e1002014 (2011).

17. C. Y. Hsu et al., FLOWERING LOCUS T duplication coordinates reproductive and vegetative growth in perennial poplar. Proc. Natl. Acad. Sci. U.S.A. 108, 10756-10761 (2011).

18. A. Azeez, P. Miskolczi, S. Tylewicz, R. P. Bhalerao, A Tree Ortholog of APETALA1 Mediates Photoperiodic Control of Seasonal Growth. Curr. Biol. 24, 717-724 (2014).

19. M. Karimi, A. Depicker, P. Hilson, Recombinational cloning with plant gateway vectors. Plant Physiol. 145, 1144-1154 (2007).

20. A. Petterle, "ABA and chromatin remodelling regulate the activity-dormancy cycle in hybrid aspen”, thesis. (Swedish University of Agricultural Sciences, Uppsala, Sweden, 2011).

21. C. Koncz, J. Schell, The promoter of TL-DNA gene 5 controls the tissue-specific expression of chimaeric genes carried by a novel type of Agrobacterium binary vector. MGG Molecular \& General Genetics 204, 383-396 (1986). 
22. O. Nilsson et al., Spatial pattern of cauliflower mosaic virus $35 \mathrm{~S}$ promoter-luciferase expression in transgenic hybrid aspen trees monitored by enzymatic assay and non-destructive imaging. Transgenic Research 1, 209-220 (1992).

23. J. Vandesompele et al., Accurate normalization of real-time quantitative RT-PCR data by geometric averaging of multiple internal control genes. Genome Biol 3, RESEARCH0034 (2002).

24. A. Karlberg, L. Bako, R. P. Bhalerao, Short day-mediated cessation of growth requires the downregulation of AINTEGUMENTALIKE1 transcription factor in hybrid aspen. PLoS Genet. 7, (2011).

25. G. A. Tuskan et al., The genome of black cottonwood, Populus trichocarpa (Torr. \& Gray). Science 313, 1596-1604 (2006).

26. N. Delhomme et al., Guidelines for RNA-Seq data analysis. Epigenesys Protocols (prot 67), (2014).

27. E. Kopylova, L. Noe, H. Touzet, SortMeRNA: fast and accurate filtering of ribosomal RNAs in metatranscriptomic data. Bioinformatics 28, 3211-3217 (2012).

28. A. M. Bolger, M. Lohse, B. Usadel, Trimmomatic: a flexible trimmer for Illumina sequence data. Bioinformatics 30, 2114-2120 (2014).

29. A. Dobin et al., STAR: ultrafast universal RNA-seq aligner. Bioinformatics 29, 15-21 (2013).

30. S. Anders, P. T. Pyl, W. Huber, HTSeq--a Python framework to work with high-throughput sequencing data. Bioinformatics 31, 166-169 (2015).

31. M. I. Love, W. Huber, S. Anders, Moderated estimation of fold change and dispersion for RNAseq data with DESeq2. Genome Biol 15, 550 (2014). 
32. S. Tylewicz et al., Dual role of tree florigen activation complex component FD in photoperiodic growth control and adaptive response pathways. Proc. Natl. Acad. Sci. U.S.A. 112, 3140-3145 (2015).

33. J. H. Venable, R. Coggeshall, A Simplified Lead Citrate Stain for Use in Electron Microscopy. J. Cell Biol. 25, 407-408 (1965).

34. P. L. H. Rinne, C. Van der Schoot, Symplasmic fields in the tunica of the shoot apical meristem coordinate morphogenetic events. Development 125, 1477-1485 (1998).

35. K. Nieminen et al., Cytokinin signaling regulates cambial development in poplar. Proc. Natl. Acad. Sci. U.S.A. 105, 20032-20037 (2008).

Acknowledgments: Funding: Grants from Vetenskapsrådet (VR-2016-04430) and Knut and Alice Wallenberg Foundation (2014-0032) to RPB are gratefully acknowledged. Author contributions: ST, AP, SM, PM, AA, RKS, JI performed experiments. NM and TRH performed the transcriptomics analysis. RPB, DME, JLB, YH, designed experiments and provided intellectual input. All authors contributed to writing the manuscript. Competing interests: The authors declare no competing interests. Data and materials availability: Raw RNA-seq reads are available at the European Nucleotide Archive (http://www.ebi.ac.uk/ena/) under accession number PRJEB23073. All other data needed to evaluate the conclusions in the paper are present in the paper or the Supplementary Materials.

\section{Supplementary Materials:}

Included in PDF file:

Materials and Methods

Figs. S1 to S8

Captions for table S1 in Excel file

Tables $\mathrm{S} 2$ to $\mathrm{S} 3$ 
Included as additional file:

Table S1

References (19-35)

Fig. 1. Hybrid aspen plants with attenuated ABA responses fail to establish dormancy. Buds of wild-type (A), abil-1 lines 1 (B) and 3 (C) after 11 weeks of short photoperiod. Unlike in WT (D), buds burst in abil-1 lines 1 (E) and 3 (F). TEM micrographs of apices of actively growing WT plants $(\mathrm{G})$ and abil-1 lines $1(\mathrm{H})$ and 3 (I) showing plasmodesmata lacking electron-dense dormancy sphincters. Sphincters are observed after 5 and 10 weeks of short photoperiod in apices of wild-type plants (J, M) (indicated with white arrows), but not abil-1 lines $1(\mathrm{~K}, \mathrm{~N})$ and $3(\mathrm{~L}, \mathrm{O})$. Bar=200 nm.

Fig. 2. PDLP1 expression restores bud dormancy in abil-1 plants. Buds of abil-1 (A) and abi1-1/PDLP1 lines 1 (B) and 3 (C) after 11 weeks of short photoperiod. Transfer to long photoperiod results in bud burst in abil-1 plants (D), but not in abil-1/PDLP1 lines 1 (E) and 3 (F).

\section{Fig. 3. $P K L$ downregulation restores dormancy sphincters and bud dormancy in abil-1} plants. TEM micrographs of apices of actively growing abil-1 plants (A) and abil-1/PKLRNAi lines 9 (B) and 11 (C) showing plasmodesmata lacking electron-dense dormancy sphincters. After 5 and 10 weeks of short photoperiod, sphincters were not observed in abil-1 apices (D, G), but were present in abil-1/PKLRNAi apices of lines $9(\mathrm{E}, \mathrm{H})$ and $11(\mathrm{~F}, \mathrm{I})$ (arrows). Bar=500 nm. Buds of abil-1 plants (J) and abil-1/PKLRNAi lines $9(\mathrm{~K})$ and 11 (L) after 11 weeks of 
short photoperiod. Following shift to long photoperiod, buds burst in abil-1 plants (M), but not in abi1-1/PKLRNAi lines $9(\mathrm{~N})$ and $11(\mathrm{O})$.

\section{Fig. 4. FT1-expressing stocks can reactivate growth in abil-1 scions under short}

photoperiod. Wild-type (WT) and abil-1 buds after 10 weeks of short photoperiod before grafting, and a further 2 and 7 weeks of short photoperiod following grafting of WT and abil-1 scions on FT1-expressing stocks. Buds remained dormant in WT scions, but burst in abil-1 scions. 


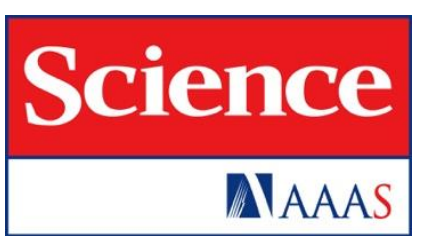

\section{Supplementary Materials for}

Photoperiodic Control of Seasonal Growth is Mediated by ABA Acting on Cell-Cell Communication

S. Tylewicz, A. Petterle, S. Marttila, P. Miskolczi, A. Azeez, R. K. Singh, J. Immanen, N. Mähler, T. R. Hvidsten, D. M. Eklund, J. L. Bowman, Y. Helariutta, R. P. Bhalerao

Correspondence to: rishi.bhalerao@slu.se

\section{Supplement:}

Included in PDF file:

Materials and Methods

Figs. S1 to S8

Captions for table $\mathrm{S} 1$ in Excel file

Tables S2 to S3

Included as additional file:

Table $\mathrm{S} 1$

References (19-35) 


\section{Materials and Methods}

\section{Plant material and growth conditions}

Hybrid aspen (Populus tremula $x$ tremuloides) clone T89 (wild type) and the transgenic plants were cultivated in half-strength MS medium (Duchefa) under sterile conditions for 5 weeks and then transferred to soil and grown for another 4 weeks in the greenhouse (16 hour light/ 8 hour dark cycles, $22^{\circ} \mathrm{C}$ and $66 \%$ relative humidity) prior to analysis of bud dormancy. For dormancy assessment, plants were shifted from the greenhouse to controlled growth chambers and grown under long photoperiod (16 hour, $20^{\circ} \mathrm{C}$ light $/ 8$ hour, $15^{\circ} \mathrm{C}$ cycles) for 1 week for acclimatization followed by exposure to 11 weeks of short photoperiod ( 8 hour, $20^{\circ} \mathrm{C}$ light $/ 16$ hour, $15^{\circ} \mathrm{C}$ dark cycles). Metal halide bulbs (HQI-T 400W daylight) were used as light source producing approximately $220 \mu \mathrm{mol} \mathrm{m}-2 \mathrm{~s}-1$ radiation. Following short photoperiod treatment, plants were shifted back to long photoperiod conditions and bud burst was monitored for at least 3 months to assess the establishment of bud dormancy (5). Shoot apices were collected for gene expression analyses after 0,6 and 10 weeks of short photoperiod, at the same time of day (14:00 hours) and stored at $-80{ }^{\circ} \mathrm{C}$ until further use. For analysis of $\mathrm{ABA}$ responsive gene expression, apices (after removal of any leaves separated from the apex) were cut and placed in MS medium with or without 50 micromolar ABA in MS medium for 4 hours with gentle shaking followed by rinsing with fresh MS and freezing until isolation of RNA. Three pools of three apices from three independent plants were collected for every sample providing three biological replicates. Photographs of apices were taken using a Canon EOS digital camera to monitor bud formation.

\section{Generation of plasmids}

To generate the PDLP1-GFP construct, a fragment containing PDLP1-GFP was amplified from plasmid (a kind gift from Prof. Maule, John Innes Institute, UK) using PDLP-For 5'-CACCGGATCCATGAAACTCACCTATCAAT-3' and EGFP-BamRev 5'-TTTTTGGATCCCTACTTGTACAGCTCGT-3' primers. This fragment was cloned into pENTR/D-TOPO (Invitrogen) and subsequently transferred into the pH2GW7 plant transformation vector to generate a plasmid designated pH2GW7-PDLP1-GFP. To generate the PKLRNAi construct, a 346 bp fragment was amplified from hybrid aspen cDNA using 5'-CACCCAGATGATATTATACTGCGCTACCC-3' and 5'TAACAGTGCTGGGTTTGCAG-3' primers. This fragment was then cloned into pENTR/D-TOPO and subsequently transferred into pK7GWIWG2 (I) (19).

\section{Plant transformation}

The generation of hybrid aspen plants expressing abil-1 has been previously described (20). The transformation constructs were introduced into Agrobacterium strain GV3101pmp90RK (21), then used to transform hybrid aspen clone T89 as previously described (22). The vector $\mathrm{pH} 2 \mathrm{GW} 7-\mathrm{PDLP} 1-\mathrm{GFP}$ was used to transform abil-1 plants to generate $P D L P 1-G F P$-overexpression in the abil-1 background. The vector pK7GWIWG2 (I)-PKLRNAi was used to transform abil-1 plants to generate PKLRNAi overexpression in the abil-1 background. 


\section{RNA isolation and quantitative real-time PCR (qRT-PCR) analysis}

Total RNA was extracted, using an Aurum Total RNA kit (Bio-Rad) or RNeasy® Plus Universal Kit (Qiagen), from tissue samples taken at the same time of day. Portions $(10 \mu \mathrm{g})$ of total RNA were treated with RNase-Free DNase (Qiagen) and cleaned using an RNeasy® Mini Kit (Qiagen). One $\mu \mathrm{g}$ of the RNA from each sample was used to generate cDNA using an iScript cDNA synthesis kit (BioRad). Selected (UBQ/TIP41like) reference genes were validated using GeNorm Software (23). qRT-PCR analyses were carried out with a Roche LightCycler 480 II instrument and relative expression values were calculated using the $\Delta$-ct-method, as previously described (24).

\section{RNA sequencing and analysis}

RNAseq was performed at BGI (Beijing, China) using RNA isolated from apices of wild type and abil-1/line 1 at 0, 6 and 10 weeks long photoperiod. RNA-Seq reads were mapped to version 3 of the Populus trichocarpa genome (25) using the approach described earlier (26). In short, ribosomal RNA was filtered using sortmerna (27) and consequently trimmed using trimmomatic (28) before being mapped to the genome using STAR (29). Reads per gene were counted using htseq-count from HTSeq (30) and differential expression analysis was performed on raw counts using DESeq2 (31). Genes with a Benjamini-Hochberg adjusted p-value $<0.05$ and an absolute $\log _{2}$ fold change $>1$ were considered differentially expressed. For further analysis of poplar homologs of Arabidopsis plasmodesmata enriched genes, an adjusted p-value $<0.05$ and an absolute $\log 2$ fold change $>0.2$ were used as filtering criteria. Raw RNA-seq reads are available at the European Nucleotide Archive (http://www.ebi.ac.uk/ena/) under accession number PRJEB23073.

\section{Western blot analysis}

Total protein was extracted from leaves of untransformed control and two independent PDLP1-GFP transformants. Western blotting was performed as described earlier (32). PDLP1-GFP levels were analyzed using anti-GFP antibodies (Novus Biologicals).

\section{Transmission electron microscopy}

Hybrid aspen buds and actively growing apices collected after 0, 5 and 10 weeks of short photoperiod were fixed in $2 \%$ glutaraldehyde and $2 \%$ paraformaldehyde in $0.02 \mathrm{M}$ cacodylate buffer ( $\mathrm{pH} 7.2$ ), combined with $1 \%$ tannic acid; postfixed $1 \% \mathrm{OsO}_{4}$ in water, dehydrated, infiltrated and embedded in Spurr's resin (Ted Pella). Ultra-thin sections were stained with uranyl acetate and lead citrate (33), and examined in transmission electron microscope (JEOL). For quantitative analysis, dormancy sphincters, visualized as electron-dense dots and marking closed plasmodesmata, were counted as percentage of the total number of counted plasmodesmata (34). In each experiment in which dormancy sphincters were examined, these were counted in samples from three independent plants representing each genotype and timepoint combination. Results are reported as percentage plasmodesmata with closed plasmodesmata. 


\section{Grafting experiments}

Soil-grown wild type and abil-1 plants were exposed to short photoperiod conditions for 10 weeks as already described. Apices of wild type and abil-1 plants were grafted onto $F T 1$-overexpressing plants, as previously reported (35), then the grafted plants were returned to the short photoperiod chamber and monitored for bud break. 


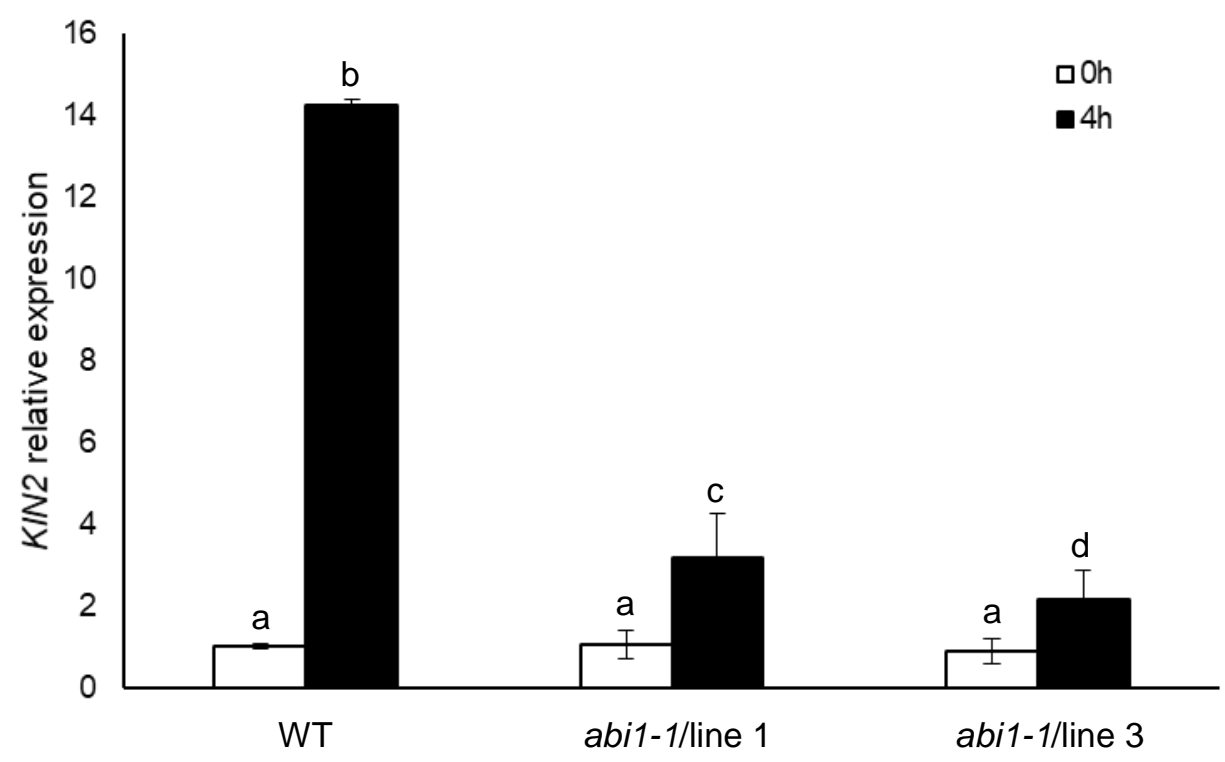

Fig. S1

Expression of abil-1 in hybrid aspen leads to attenuation of ABA responses. Relative transcript levels of KIN2 before and after ABA treatment of wild type (WT) and abil-1 plants (line 1 and line 3 ). Bars show the average of three biological replicates \pm standard error $(\mathrm{SE})$. Letters $(\mathrm{a}-\mathrm{d})$ over the bars indicate significant differences at $\mathrm{P}<0.05$ (means followed by the same letter are not significantly different at $\mathrm{P}<0.05$ ). 

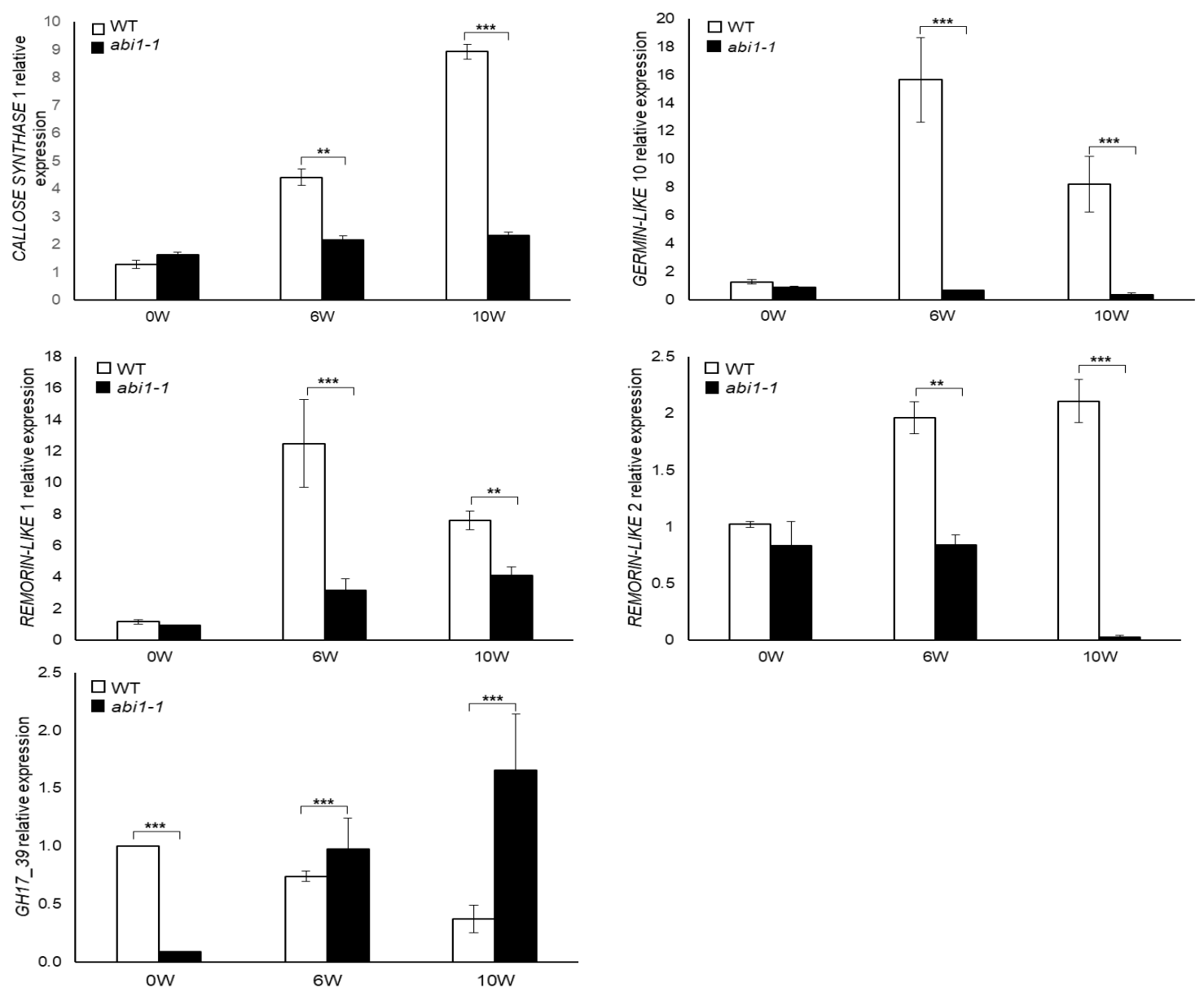

Fig. S2

Relative expression of plasmodesmata-associated marker genes in apices of wild-type and abil-1 (line 1) plants before (0W) and after 6 and 10 weeks (6W and 10W) of short photoperiod treatment. Bars show the average of three biological replicates $\pm \mathrm{SE}$. Asterisks $(* *)$ indicate very significant differences at $\mathrm{P}<0.01$ and $* * *$ extremely significant $(\mathrm{P}<0.001)$ with respect to $\mathrm{WT}$ at each time point. 


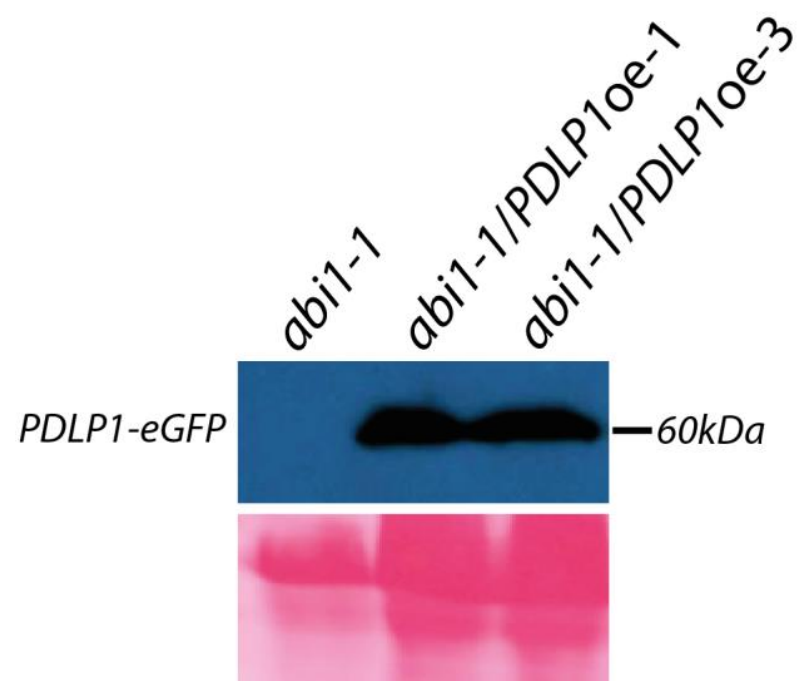

\section{Fig. S3}

Western blot analysis of PDLP1-eGFP levels in abil-1 apices (controls) and two independent PDLP1-eGFP transformed abil-1 lines (upper panel). The lower panel shows the same blot stained with Ponceau S. 


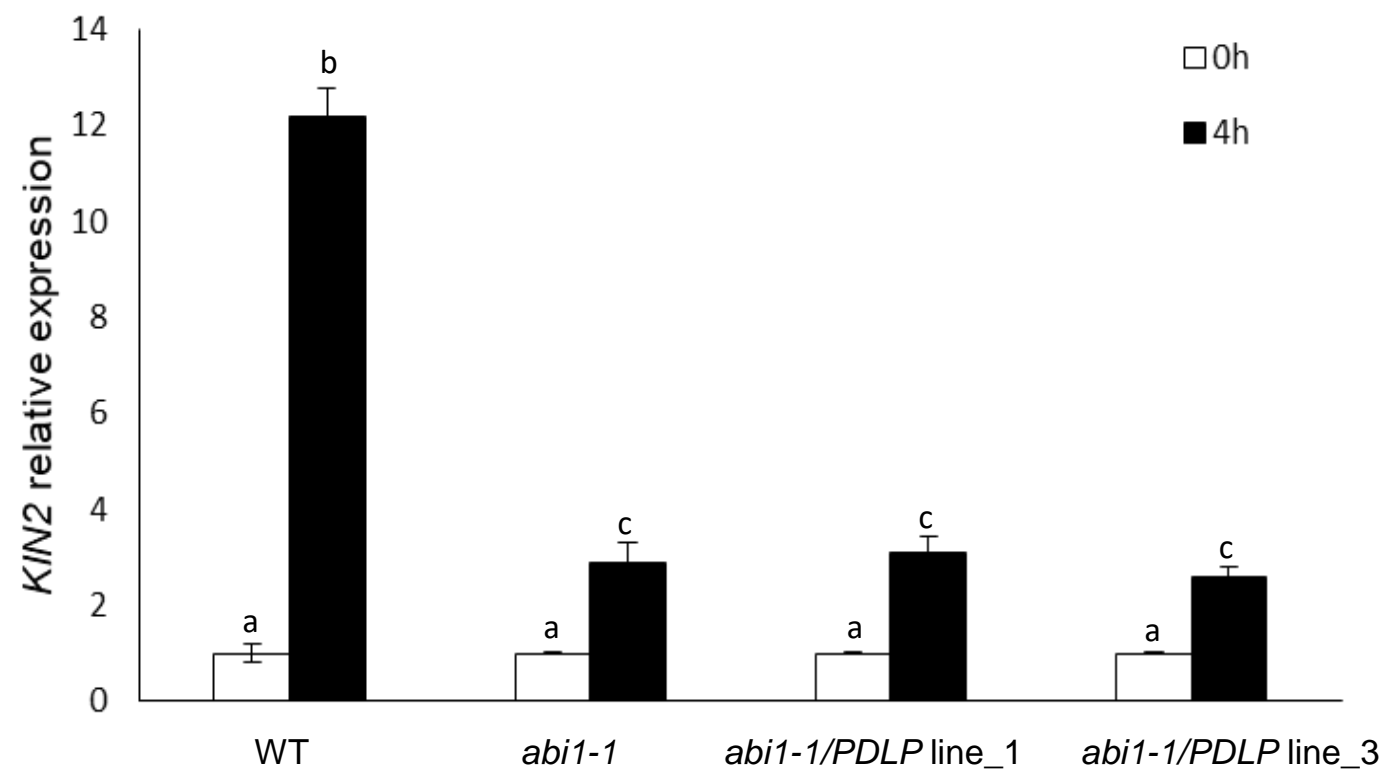

Fig. $\mathbf{S 4}$

Expression of PDLP in abil-1 does not leads to attenuation of ABA responses.

Relative transcript levels of KIN2 before and after ABA treatment of wild type (WT), abil-1 plants and abil-1 plants expressing PDLP (abil-1/PDLP lines 1 and line 3). Bars show the average of three biological replicates \pm SE. Letters $(a-c)$ over the bars indicate significant differences at $\mathrm{P}<0.05$ (means followed by the same letter are not significantly different at $\mathrm{P}<0.05)$. 


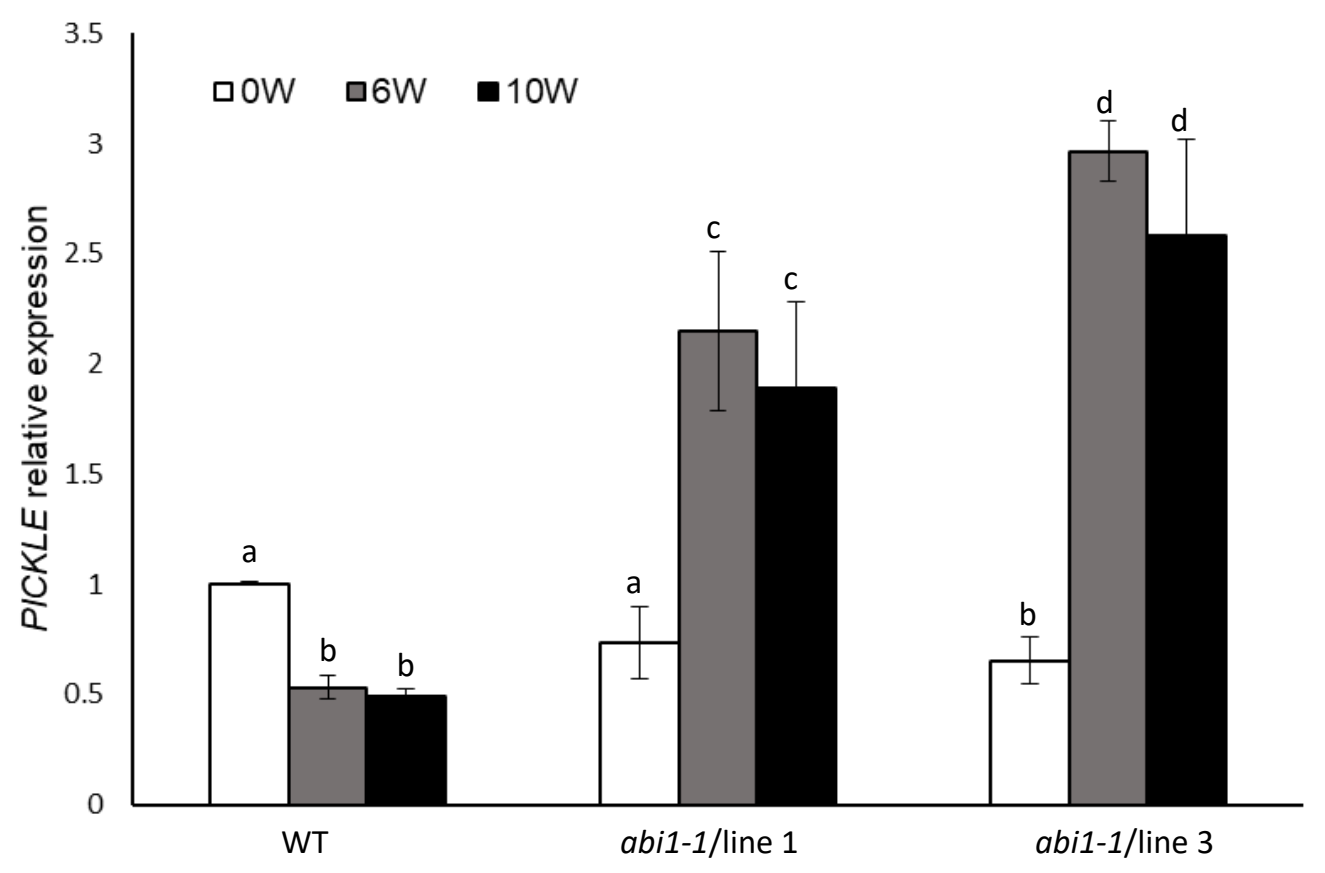

Fig. S5

Altered $P K L$ expression in abil-1 plants after short photoperiod. $P K L$ transcript levels are reduced in WT but increased in abil-1 plants after short photoperiod. The graph shows relative levels of $P K L$ in WT and abil-1 plants before (0W) and after 6 and 10 weeks $(6 \mathrm{~W}$ and $10 \mathrm{~W}$ ) of short photoperiod treatment. Bars show the average of three biological replicates \pm SE. Letters $(a-d)$ over the bars indicate significant differences at $\mathrm{P}<0.05$ (means followed by the same letter are not significantly different at $\mathrm{P}<0.05$ ). 


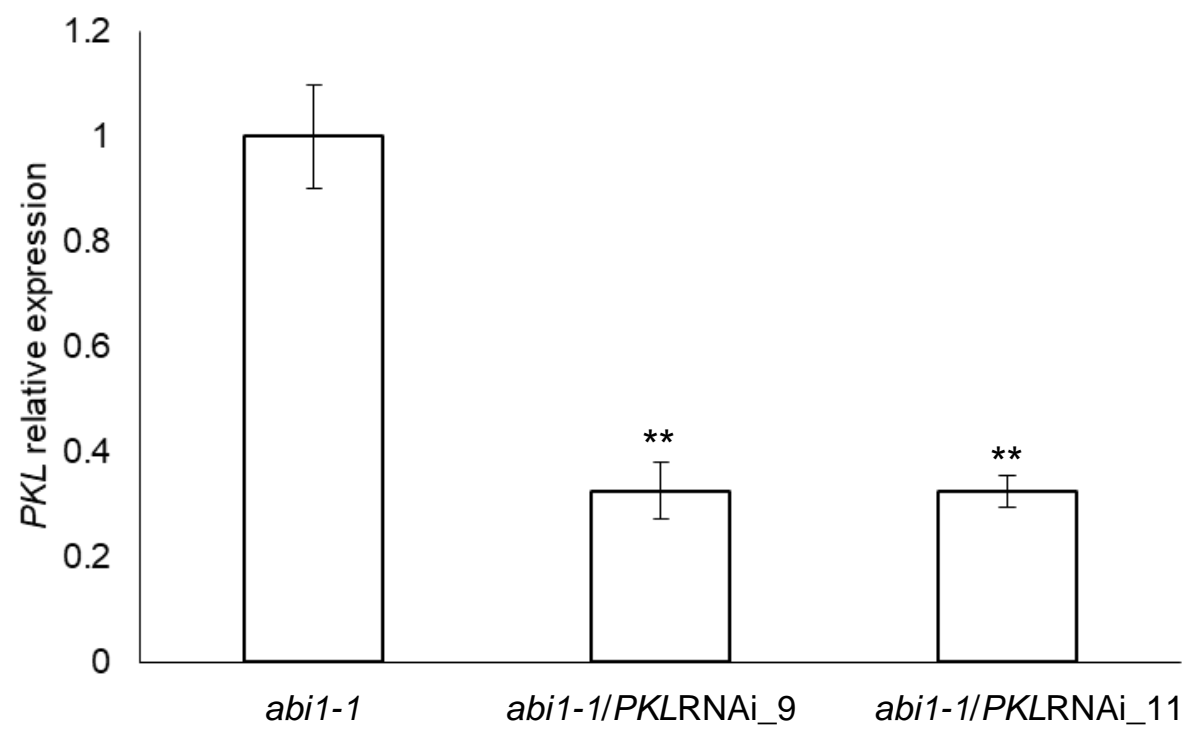

\section{Fig. S6}

$P K L$ transcript levels are reduced in abil-1/PKLRNAi plants. The graph shows relative levels of $P K L$ in abil-1 and abil-1/PKLRNAi lines. Bars show the average of three biological replicates \pm SE. Asterisk (**) indicate a significant difference at $\mathrm{P}<0.01$ with respect to control (abil-1) plants. 

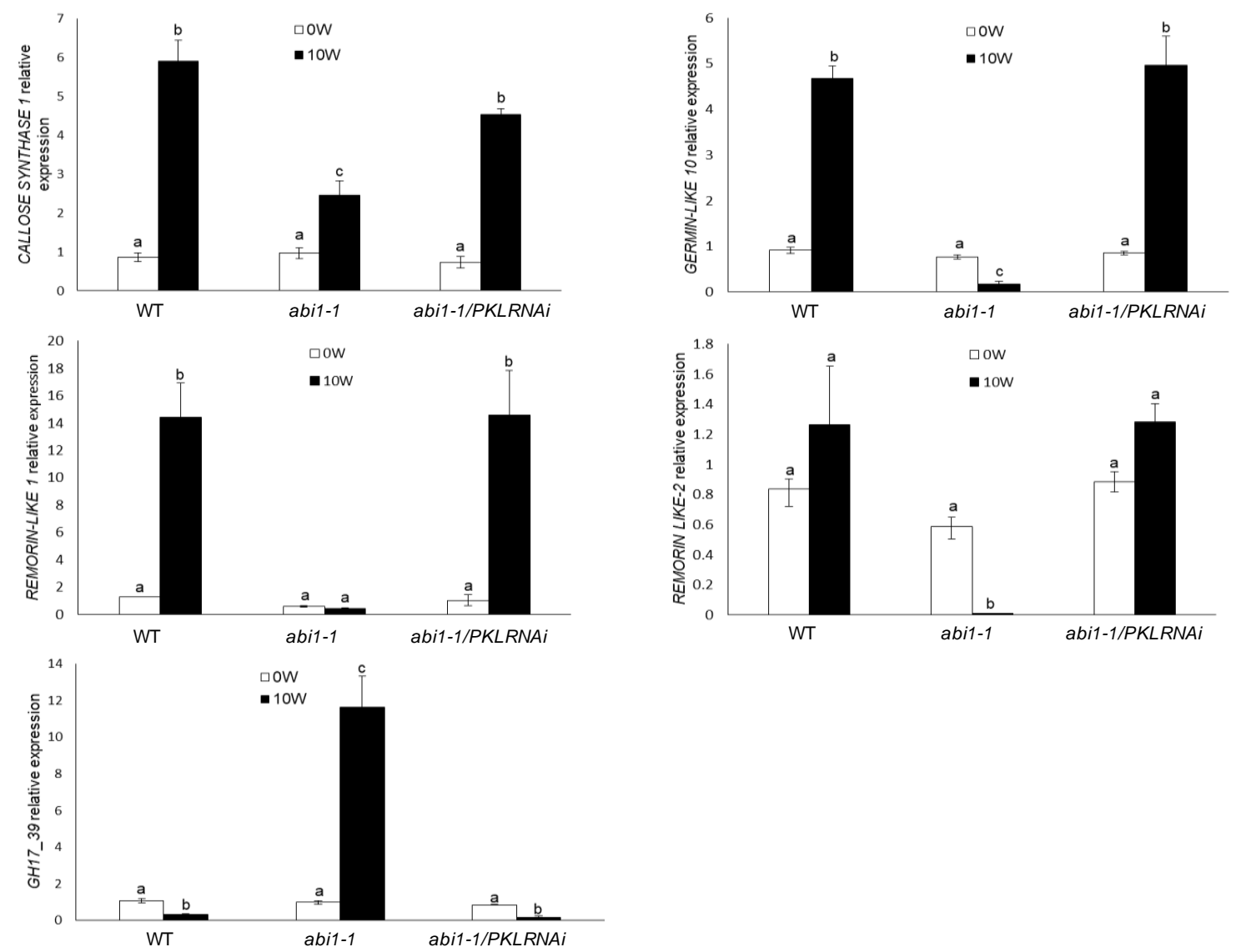

Fig. S7

Relative expression levels of plasmodesmata-associated marker genes in apices of wildtype, abil-1 (line 1) and abil-1/PKLRNAi plants before (0W) and after 10 week (10W) of short photoperiod treatment. Bars show the average of three biological replicates $\pm \mathrm{SE}$. Letters $(\mathrm{a}-\mathrm{c})$ over the bars indicate significant differences at $\mathrm{P}<0.05$ (means followed by the same letter are not significantly different at $\mathrm{P}<0.05)$. 


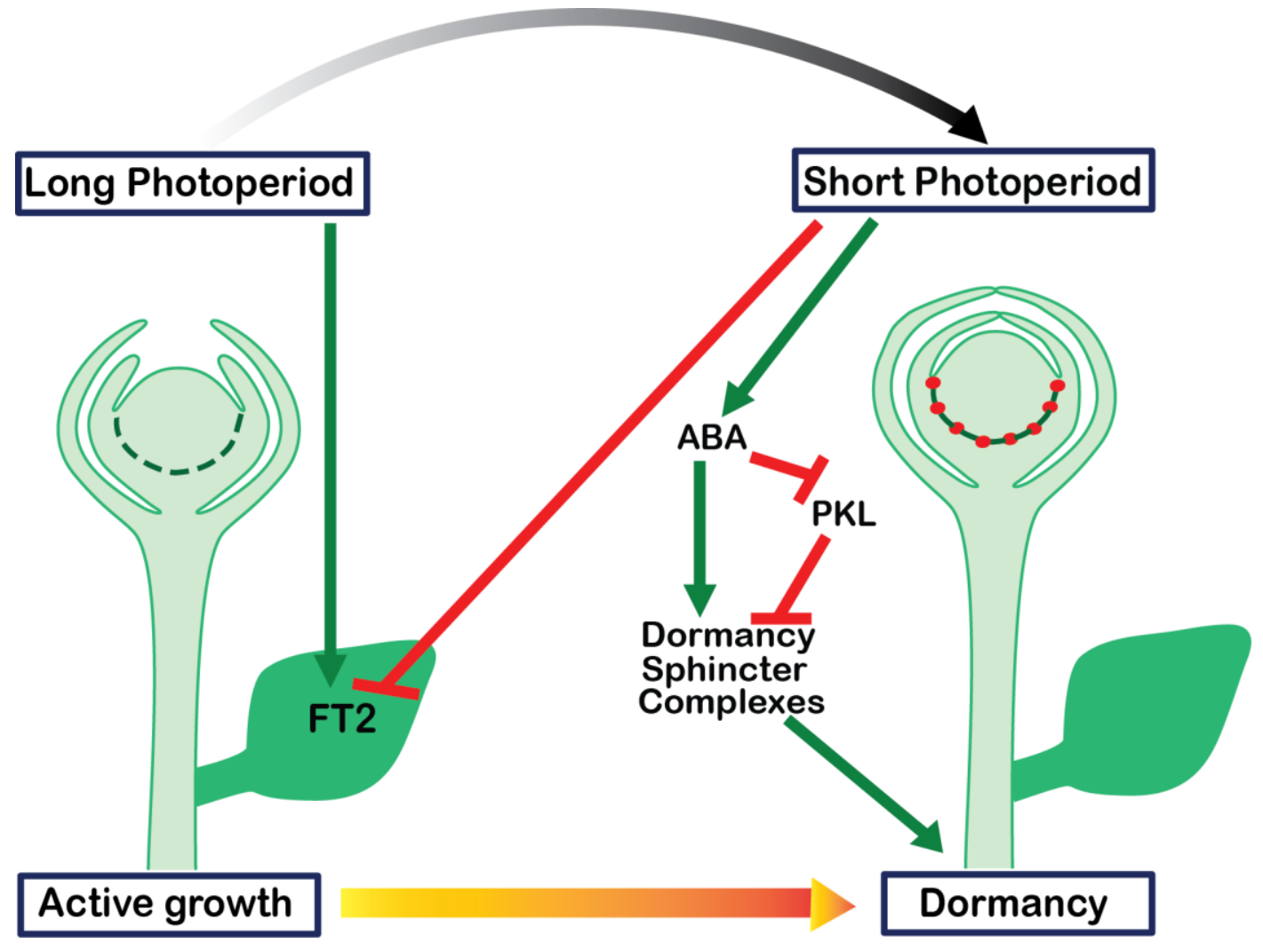

Fig. S8

Schematic model of photoperiodic control of bud dormancy. In long photoperiod, plasmodesmata are open, allowing SAM access to growth-promotive signals, e.g. FT, permitting growth. Short photoperiod suppress $F T$ expression and downregulate $P K L$, inducing ABA-mediated formation of dormancy sphincters, limiting access of SAM and promoting dormancy. 


\section{Table S1 caption:}

Differential expression results. Each sheet contains results for one comparison e.g. WT_6w_vs_0w contains comparisons of gene expression between 6 weeks and 0 weeks of short photoperiod treatment in the wild-type. Columns A-C contain gene information from Phytozome (https://phytozome.jgi.doe.gov). Column D (baseMean): mean expression over e.g. all 6 weeks and 0 weeks replicates. Column E (log2FoldChange): $\log 2$ fold change expression between e.g. 6 weeks and 0 weeks of short photoperiod treatment. Column F (lfcSE): standard error of the $\log 2$ fold change. Column G (pvalue): p-value for the comparison. Column $\mathrm{H}$ (padj): Benjamini-Hochberg adjusted p-value. 


\section{Table S2}

List of Populus trichocarpa genes that are homologous to the genes that were identified in the plasmodesmata fraction of Arabidopsis thaliana, based on best BLAST hits. Columns contain gene information from PopGenIE and AtGenIE (http://popgenie.org/, http://atgenie.org/ ).

\begin{tabular}{|c|c|c|c|}
\hline $\begin{array}{l}\text { P. trichocarpa } \\
\text { gene ID }\end{array}$ & At gene ID & Description in Atgenie & At synonyms \\
\hline Potri.001G065900 & AT1G28110 & serine carboxypeptidase-like 45 & SCPL45 \\
\hline Potri.001G092200 & AT1G64390 & glycosyl hydrolase $9 \mathrm{C2}$ & AtGH9C2,GH9C2 \\
\hline Potri.001G108700 & AT1G63120 & RHOMBOID-like 2 & ATRBL2,RBL2 \\
\hline Potri.001G112100 & AT2G42010 & phospholipase $\mathrm{D}$ beta 1 & PLDBETA,PLDBETA1 \\
\hline Potri.001G129300 & AT4G28050 & tetraspanin7 & TET7 \\
\hline Potri.001G141400 & AT5G46700 & Tetraspanin family protein & TET1,TRN2 \\
\hline Potri.001G200400 & AT3G13750 & beta galactosidase 1 & BGAL1 \\
\hline Potri.001G236400 & AT3G46010 & actin depolymerizing factor 1 & ADF1,atadf,ATADF1 \\
\hline Potri.001G240900 & AT2G39700 & expansin A4 & \begin{tabular}{|l|} 
ATEXP4,ATEXPA4,ATHEXP ALPHA \\
1.6,EXPA4 \\
\end{tabular} \\
\hline Potri.001G278300 & AT5G58600 & Plant protein of unknown function (DUF828) & PMR5,TBL44 \\
\hline Potri.001G283000 & AT1G74520 & HVA22 homologue $\mathrm{A}$ & ATHVA22A,HVA22A \\
\hline Potri.001G311700 & AT5G64260 & EXORDIUM like 2 & EXL2 \\
\hline Potri.001G342200 & AT5G14420 & RING domain ligase2 & RGLG2 \\
\hline Potri.001G357800 & AT1G30460 & cleavage and polyadenylation specificity factor 30 & ATCPSF30,CPSF30 \\
\hline Potri.001G423700 & AT3G20500 & purple acid phosphatase 18 & ATPAP18,PAP18 \\
\hline Potri.001G442400 & AT4G20380 & LSD1 zinc finger family protein & LSD1 \\
\hline Potri.002G029300 & AT2G16430 & purple acid phosphatase 10 & ATPAP10,PAP10 \\
\hline Potri.002G060400 & AT4G14130 & xyloglucan endotransglucosylase/hydrolase 15 & XTH15,XTR7 \\
\hline Potri.002G060500 & AT4G14130 & xyloglucan endotransglucosylase/hydrolase 15 & XTH15,XTR7 \\
\hline Potri.002G098900 & AT5G64260 & EXORDIUM like 2 & EXL2 \\
\hline Potri.002G120600 & AT4G24220 & NAD(P)-binding Rossmann-fold superfamily protein & AWI31,VEP1 \\
\hline Potri.002G120700 & AT4G24220 & NAD(P)-binding Rossmann-fold superfamily protein & AWI31,VEP1 \\
\hline Potri.002G129400 & AT2G26510 & Xanthine/uracil permease family protein & PDE135 \\
\hline Potri.002G145400 & AT3G60720 & plasmodesmata-located protein 8 & \begin{tabular}{|l|l|} 
PDLP8 \\
\end{tabular} \\
\hline Potri.002G152100 & AT2G42010 & phospholipase D beta 1 & PLDBETA,PLDBETA1 \\
\hline Potri.002G200300 & AT1G02730 & cellulose synthase-like D5 & ATCSLD5,CSLD5,SOS6 \\
\hline Potri.002G257300 & AT1G04520 & plasmodesmata-located protein 2 & PDLP2 \\
\hline Potri.003G038500 & AT3G13750 & beta galactosidase 1 & BGAL1 \\
\hline Potri.003G093000 & AT5G46700 & Tetraspanin family protein & TET1,TRN2 \\
\hline Potri.003G122900 & AT1G63120 & RHOMBOID-like 2 & ATRBL2,RBL2 \\
\hline Potri.003G134500 & AT4G24220 & NAD(P)-binding Rossmann-fold superfamily protein & AWI31,VEP1 \\
\hline Potri.003G139600 & AT1G64390 & glycosyl hydrolase 9C2 & AtGH9C2,GH9C2 \\
\hline Potri.003G164000 & AT1G28110 & serine carboxypeptidase-like 45 & SCPL45 \\
\hline Potri.004G010100 & AT4G22010 & SKU5 similar 4 & sks4 \\
\hline Potri.004G042400 & AT4G25810 & xyloglucan endotransglycosylase 6 & XTH23,XTR6 \\
\hline Potri.004G100700 & AT3G02540 & Rad23 UV excision repair protein family & RAD23-3,RAD23C \\
\hline Potri.004G123200 & AT1G69530 & expansin A1 & $\begin{array}{l}\text { AT-EXP1,ATEXP1,ATEXPA1,ATHEXP } \\
\text { ALPHA 1.2,EXP1,EXPA1 }\end{array}$ \\
\hline Potri.004G160100 & AT2G16430 & purple acid phosphatase 10 & ATPAP10,PAP10 \\
\hline Potri.004G160200 & AT2G16430 & purple acid phosphatase 10 & ATPAP10,PAP10 \\
\hline Potri.004G173900 & AT4G34980 & subtilisin-like serine protease 2 & SLP2 \\
\hline Potri.004G184500 & AT2G27600 & AAA-type ATPase family protein & ATSKD1,SKD1,VPS4 \\
\hline Potri.004G206600 & AT2G17230 & EXORDIUM like 5 & EXL5 \\
\hline Potri.005G000100 & AT5G26742 & DEAD box RNA helicase (RH3) & emb1138 \\
\hline Potri.005G000200 & AT5G26742 & DEAD box RNA helicase (RH3) & emb1138 \\
\hline Potri.005G000500 & AT5G26742 & DEAD box RNA helicase (RH3) & emb1138 \\
\hline Potri.005G007200 & AT4G25810 & xyloglucan endotransglycosylase 6 & XTH23,XTR6 \\
\hline Potri.005G128800 & AT4G36860 & LIM domain-containing protein & DAR1 \\
\hline Potri.005G128900 & AT4G36860 & LIM domain-containing protein & DAR1 \\
\hline Potri.005G152100 & AT1G69700 & HVA22 homologue C & ATHVA22C,HVA22C \\
\hline
\end{tabular}




\begin{tabular}{|c|c|c|c|}
\hline Potri.005G163000 & AT5G64260 & EXORDIUM like 2 & EXL2 \\
\hline Potri.005G201200 & AT4G14130 & xyloglucan endotransglucosylase/hydrolase 15 & XTH15,XTR7 \\
\hline Potri.005G233400 & AT2G27190 & purple acid phosphatase 12 & ATPAP1,ATPAP12,PAP1,PAP12 \\
\hline Potri.006G002600 & AT5G14420 & RING domain ligase2 & RGLG2 \\
\hline Potri.006G003600 & AT1G60870 & maternal effect embryo arrest 9 & MEE9 \\
\hline Potri.006G006100 & AT5G63530 & farnesylated protein 3 & ATFP3,FP3 \\
\hline Potri.006G032100 & AT2G40830 & RING-H2 finger C1A & RHC1A \\
\hline Potri.006G034200 & AT3G56640 & exocyst complex component sec15A & SEC15A \\
\hline Potri.006G036000 & AT1G28110 & serine carboxypeptidase-like 45 & SCPL45 \\
\hline Potri.006G036400 & AT2G33530 & serine carboxypeptidase-like 46 & scpl46 \\
\hline Potri.006G036500 & AT1G28110 & serine carboxypeptidase-like 45 & SCPL45 \\
\hline Potri.006G058500 & AT5G06860 & polygalacturonase inhibiting protein 1 & ATPGIP1,PGIP1 \\
\hline Potri.006G058600 & AT5G06860 & polygalacturonase inhibiting protein 1 & ATPGIP1,PGIP1 \\
\hline Potri.006G071200 & AT4G25810 & xyloglucan endotransglycosylase 6 & XTH23,XTR6 \\
\hline Potri.006G098700 & AT1G74720 & $\begin{array}{l}\text { C2 calcium/lipid-binding plant phosphoribosyltransferase } \\
\text { family protein }\end{array}$ & QKY \\
\hline Potri.006G144500 & AT3G13750 & beta galactosidase 1 & BGAL1 \\
\hline Potri.006G150400 & AT2G19580 & tetraspanin2 & TET2 \\
\hline Potri.006G169900 & AT4G25810 & xyloglucan endotransglycosylase 6 & XTH23,XTR6 \\
\hline Potri.006G170000 & AT4G25810 & xyloglucan endotransglycosylase 6 & XTH23,XTR6 \\
\hline Potri.006G170100 & AT4G25810 & xyloglucan endotransglycosylase 6 & XTH23,XTR6 \\
\hline Potri.006G177900 & AT2G23810 & tetraspanin8 & TET8 \\
\hline Potri.006G204200 & AT3G11660 & NDR1/HIN1-like 1 & NHL1 \\
\hline Potri.007G032500 & AT4G36860 & LIM domain-containing protein & DAR1 \\
\hline Potri.007G083400 & AT3G45970 & expansin-like A1 & $\begin{array}{l}\text { ATEXLA1,ATEXPL1,ATHEXP BETA } \\
\text { 2.1,EXLA1,EXPL1 }\end{array}$ \\
\hline Potri.008G010900 & AT5G04990 & SAD1/UNC-84 domain protein 1 & ATSUN1,SUN1 \\
\hline Potri.008G018300 & AT5G04850 & SNF7 family protein & VPS60.2 \\
\hline Potri.008G022300 & AT2G27600 & AAA-type ATPase family protein & ATSKD1,SKD1,VPS4 \\
\hline Potri.008G032100 & AT5G48450 & SKU5 similar 3 & sks3 \\
\hline Potri.008G038100 & AT5G03300 & adenosine kinase 2 & ADK2 \\
\hline Potri.008G057100 & AT2G39700 & expansin A4 & $\begin{array}{l}\text { ATEXP4,ATEXPA4,ATHEXP ALPHA } \\
\text { 1.6,EXPA4 }\end{array}$ \\
\hline Potri.008G088300 & AT1G69530 & expansin A1 & $\begin{array}{l}\text { AT-EXP1,ATEXP1,ATEXPA1,ATHEXP } \\
\text { ALPHA 1.2,EXP1,EXPA1 }\end{array}$ \\
\hline Potri.008G095300 & AT1G18650 & plasmodesmata callose-binding protein 3 & PDCB3 \\
\hline Potri.008G119700 & AT3G02540 & Rad23 UV excision repair protein family & RAD23-3,RAD23C \\
\hline Potri.008G133500 & AT2G01660 & plasmodesmata-located protein 6 & PDLP6 \\
\hline Potri.008G171700 & AT1G04520 & plasmodesmata-located protein 2 & PDLP2 \\
\hline Potri.008G183600 & AT5G14420 & RING domain ligase 2 & RGLG2 \\
\hline Potri.008G193700 & AT1G10510 & RNI-like superfamily protein & emb2004 \\
\hline Potri.009G015100 & AT3G45600 & tetraspanin3 & TET3 \\
\hline Potri.009G031800 & AT2G39700 & expansin A4 & $\begin{array}{l}\text { ATEXP4,ATEXPA4,ATHEXP ALPHA } \\
\text { 1.6,EXPA4 }\end{array}$ \\
\hline Potri.009G072800 & AT5G58600 & Plant protein of unknown function (DUF828) & PMR5,TBL44 \\
\hline Potri.009G078500 & AT1G74520 & HVA22 homologue $A$ & ATHVA22A,HVA22A \\
\hline Potri.009G092000 & AT4G24220 & NAD(P)-binding Rossmann-fold superfamily protein & AWI31,VEP1 \\
\hline Potri.009G100500 & AT4G34980 & subtilisin-like serine protease 2 & SLP2 \\
\hline Potri.009G121200 & AT2G16430 & purple acid phosphatase 10 & ATPAP10,PAP10 \\
\hline Potri.009G133400 & AT4G34980 & subtilisin-like serine protease 2 & SLP2 \\
\hline Potri.009G144200 & AT2G27600 & AAA-type ATPase family protein & ATSKD1,SKD1,VPS4 \\
\hline Potri.009G144300 & AT2G27600 & AAA-type ATPase family protein & ATSKD1,SKD1,VPS4 \\
\hline Potri.009G167800 & AT2G17230 & EXORDIUM like 5 & EXL5 \\
\hline Potri.010G049600 & AT5G14420 & RING domain ligase2 & RGLG2 \\
\hline Potri.010G065800 & AT1G04520 & plasmodesmata-located protein 2 & PDLP2 \\
\hline Potri.010G108100 & AT2G01660 & plasmodesmata-located protein 6 & PDLP6 \\
\hline Potri.010G114200 & AT1G71040 & Cupredoxin superfamily protein & LPR2 \\
\hline Potri.010G126200 & AT3G02540 & Rad23 UV excision repair protein family & RAD23-3,RAD23C \\
\hline Potri.010G158900 & AT1G18650 & plasmodesmata callose-binding protein 3 & PDCB3 \\
\hline Potri.010G167200 & AT1G69530 & expansin A1 & $\begin{array}{l}\text { AT-EXP1,ATEXP1,ATEXPA1,ATHEXP } \\
\text { ALPHA 1.2,EXP1,EXPA1 }\end{array}$ \\
\hline Potri.010G202500 & AT2G39700 & expansin A4 & $\begin{array}{l}\text { ATEXP4,ATEXPA4,ATHEXP ALPHA } \\
\text { 1.6,EXPA4 }\end{array}$ \\
\hline
\end{tabular}




\begin{tabular}{|c|c|c|c|}
\hline Potri.010G224300 & AT5G03300 & adenosine kinase 2 & ADK2 \\
\hline Potri.010G237100 & AT2G27600 & AAA-type ATPase family protein & ATSKD1,SKD1,VPS4 \\
\hline Potri.010G247900 & AT5G04990 & SAD1/UNC-84 domain protein 1 & ATSUN1,SUN1 \\
\hline Potri.011G052400 & AT4G04970 & glucan synthase-like 1 & ATGSL01,ATGSL1,GSL01,GSL1 \\
\hline Potri.011G077200 & AT4G18040 & eukaryotic translation initiation factor $4 \mathrm{E}$ & AT.EIF4E1,CUM1,EIF4E,elF4E1 \\
\hline Potri.011G077400 & AT4G14130 & xyloglucan endotransglucosylase/hydrolase 15 & XTH15,XTR7 \\
\hline Potri.011G089800 & AT1G30460 & cleavage and polyadenylation specificity factor 30 & ATCPSF30,CPSF30 \\
\hline Potri.011G089900 & AT1G30460 & cleavage and polyadenylation specificity factor 30 & ATCPSF30,CPSF30 \\
\hline Potri.011G135500 & AT4G27080 & PDI-like 5-4 & ATPDI7,ATPDIL5-4,PDI7,PDIL5-4 \\
\hline Potri.011G138200 & AT3G20500 & purple acid phosphatase 18 & ATPAP18,PAP18 \\
\hline Potri.011G154800 & AT4G20380 & LSD1 zinc finger family protein & LSD1 \\
\hline Potri.012G006100 & AT5G24520 & Transducin/WD40 repeat-like superfamily protein & ATTTG1,TTG,TTG1,URM23 \\
\hline Potri.012G061600 & AT5G61030 & glycine-rich RNA-binding protein 3 & GR-RBP3 \\
\hline Potri.012G065700 & AT1G18650 & plasmodesmata callose-binding protein 3 & PDCB3 \\
\hline Potri.012G069300 & AT1G74520 & HVA22 homologue $A$ & ATHVA22A,HVA22A \\
\hline Potri.012G090100 & AT5G14420 & RING domain ligase2 & RGLG2 \\
\hline Potri.012G101400 & AT5G63530 & farnesylated protein 3 & ATFP3,FP3 \\
\hline Potri.012G105500 & AT1G28110 & serine carboxypeptidase-like 45 & SCPL45 \\
\hline Potri.012G128100 & AT5G51550 & EXORDIUM like 3 & EXL3 \\
\hline Potri.013G005700 & AT4G25810 & xyloglucan endotransglycosylase 6 & XTH23,XTR6 \\
\hline Potri.013G048200 & AT1G04520 & plasmodesmata-located protein 2 & PDLP2 \\
\hline Potri.013G118500 & AT2G42570 & TRICHOME BIREFRINGENCE-LIKE 39 & TBL39 \\
\hline Potri.013G152400 & AT4G03210 & xyloglucan endotransglucosylase/hydrolase 9 & XTH9 \\
\hline Potri.014G019300 & AT4G24220 & NAD(P)-binding Rossmann-fold superfamily protein & AWI31,VEP1 \\
\hline Potri.014G019400 & AT4G24220 & NAD(P)-binding Rossmann-fold superfamily protein & AWI31,VEP1 \\
\hline Potri.014G019500 & AT4G24220 & NAD(P)-binding Rossmann-fold superfamily protein & AWI31,VEP1 \\
\hline Potri.014G019600 & AT4G24220 & NAD(P)-binding Rossmann-fold superfamily protein & AWI31,VEP1 \\
\hline Potri.014G019700 & AT4G24220 & NAD(P)-binding Rossmann-fold superfamily protein & AWI31,VEP1 \\
\hline Potri.014G035800 & AT2G26510 & Xanthine/uracil permease family protein & PDE135 \\
\hline Potri.014G067000 & AT3G60720 & plasmodesmata-located protein 8 & PDLP8 \\
\hline Potri.014G074700 & AT2G42010 & phospholipase $\mathrm{D}$ beta 1 & PLDBETA,PLDBETA1 \\
\hline Potri.014G125100 & AT1G02730 & cellulose synthase-like D5 & ATCSLD5,CSLD5,SOS6 \\
\hline Potri.014G126000 & AT5G64260 & EXORDIUM like 2 & EXL2 \\
\hline Potri.014G148600 & AT1G74520 & HVA22 homologue A & ATHVA22A,HVA22A \\
\hline Potri.014G154500 & AT4G22010 & SKU5 similar 4 & sks4 \\
\hline Potri.014G177700 & AT5G48450 & SKU5 similar 3 & sks3 \\
\hline Potri.015G002600 & AT5G24520 & Transducin/WD40 repeat-like superfamily protein & ATTTG1,TTG,TTG1,URM23 \\
\hline Potri.015G055700 & AT2G23810 & tetraspanin8 & TET8 \\
\hline Potri.015G057400 & AT5G61030 & glycine-rich RNA-binding protein 3 & GR-RBP3 \\
\hline Potri.015G057800 & AT1G18650 & plasmodesmata callose-binding protein 3 & PDCB3 \\
\hline Potri.015G062800 & AT1G74520 & HVA22 homologue A & ATHVA22A,HVA22A \\
\hline Potri.015G086200 & AT5G14420 & RING domain ligase2 & RGLG2 \\
\hline Potri.015G104700 & AT1G28110 & serine carboxypeptidase-like 45 & SCPL45 \\
\hline Potri.015G129700 & AT5G51550 & EXORDIUM like 3 & EXL3 \\
\hline Potri.016G003000 & AT5G14420 & RING domain ligase2 & RGLG2 \\
\hline Potri.016G004300 & AT1G60870 & maternal effect embryo arrest 9 & MEE9 \\
\hline Potri.016G029300 & AT2G40830 & RING-H2 finger $\mathrm{C} 1 \mathrm{~A}$ & RHC1A \\
\hline Potri.016G032100 & AT3G56640 & exocyst complex component sec15A & SEC15A \\
\hline Potri.016G034300 & AT1G28110 & serine carboxypeptidase-like 45 & SCPL45 \\
\hline Potri.016G034400 & AT1G28110 & serine carboxypeptidase-like 45 & SCPL45 \\
\hline Potri.016G034500 & AT1G28110 & serine carboxypeptidase-like 45 & SCPL45 \\
\hline Potri.016G034600 & AT2G33530 & serine carboxypeptidase-like 46 & scpl46 \\
\hline Potri.016G034700 & AT1G28110 & serine carboxypeptidase-like 45 & SCPL45 \\
\hline Potri.016G034900 & AT1G28110 & serine carboxypeptidase-like 45 & SCPL45 \\
\hline Potri.016G046100 & AT2G33330 & plasmodesmata-located protein 3 & PDLP3 \\
\hline Potri.016G049400 & AT5G06860 & polygalacturonase inhibiting protein 1 & ATPGIP1,PGIP1 \\
\hline Potri.016G049600 & AT5G06860 & polygalacturonase inhibiting protein 1 & ATPGIP1,PGIP1 \\
\hline Potri.016G071500 & AT3G11660 & NDR1/HIN1-like 1 & NHL1 \\
\hline Potri.016G097500 & AT2G39700 & expansin A4 & $\begin{array}{l}\text { ATEXP4,ATEXPA4,ATHEXP ALPHA } \\
\text { 1.6,EXPA4 }\end{array}$ \\
\hline Potri.016G113800 & AT1G74720 & $\begin{array}{l}\text { C2 calcium/lipid-binding plant phosphoribosyltransferase } \\
\text { family protein }\end{array}$ & QKY \\
\hline Potri.017G030300 & AT4G24220 & NAD(P)-binding Rossmann-fold superfamily protein & AWI31,VEP1 \\
\hline
\end{tabular}




\begin{tabular}{|l|l|l|l|} 
Potri.017G030400 & AT4G24220 & NAD(P)-binding Rossmann-fold superfamily protein & AWI31,VEP1 \\
\hline Potri.017G030600 & AT4G24220 & NAD(P)-binding Rossmann-fold superfamily protein & AWI31,VEP1 \\
\hline Potri.017G030700 & AT4G24220 & NAD(P)-binding Rossmann-fold superfamily protein & AWI31,VEP1 \\
\hline Potri.017G051700 & AT5G64260 & EXORDIUM like 2 & EXL2 \\
\hline Potri.017G102800 & AT2G40830 & RING-H2 finger C1A & RHC1A \\
\hline Potri.017G114000 & AT3G02540 & Rad23 UV excision repair protein family & RAD23-3,RAD23C \\
\hline Potri.017G139000 & AT1G74520 & HVA22 homologue A & ATHVA22A,HVA22A \\
\hline Potri.018G094900 & AT4G25810 & xyloglucan endotransglycosylase 6 & XTH23,XTR6 \\
\hline Potri.018G095100 & AT4G25810 & xyloglucan endotransglycosylase 6 & XTH23,XTR6 \\
\hline Potri.018G095200 & AT4G25810 & xyloglucan endotransglycosylase 6 & XTH23,XTR6 \\
\hline Potri.018G099800 & AT2G23810 & tetraspanin8 & TET8 \\
\hline Potri.019G045200 & AT3G45600 & tetraspanin3 & TET3 \\
\hline Potri.019G069300 & AT1G71380 & cellulase 3 & ATCEL3,ATGH9B3,CEL3 \\
\hline Potri.019G090200 & AT2G42570 & TRICHOME BIREFRINGENCE-LIKE 39 & TBL39 \\
\hline Potri.019G125000 & AT4G03210 & xyloglucan endotransglucosylase/hydrolase 9 & XTH9 \\
\hline Potri.T112400 & AT4G14130 & xyloglucan endotransglucosylase/hydrolase 15 & XTH15,XTR7 \\
\hline Potri.T121300 & AT2G17230 & EXORDIUM like 5 & EXL5 \\
\hline Potri.T121400 & AT2G17230 & EXORDIUM like 5 & EXL5 \\
\hline Potri.T162000 & AT5G61030 & glycine-rich RNA-binding protein 3 & GR-RBP3 \\
\hline Potri.T180000 & AT2G42010 & phospholipase D beta 1 & PLDBETA,PLDBETA1 \\
\hline Potri.T180200 & AT4G14130 & xyloglucan endotransglucosylase/hydrolase 15 & XTH15,XTR7 \\
\hline
\end{tabular}


Table S3

Sequences of primers used in quantitative RT-PCR

\begin{tabular}{|l|l|}
\hline$R E M O R I N-L I K E 1$ forward & GCAGATCCAACTGCTGATGA \\
\hline REMORIN-LIKE 1 reverse & CTCATTTTCCACCCATGCTT \\
\hline REMORIN-LIKE 2 forward & CGAACAGCTTTAGGCAAACC \\
\hline REMORIN-LIKE 2 reverse & TCCTGTCGTCTGCATTTGAG \\
\hline CALLOSE SYNTHASE 1 forward & TAAATAACCGTGGAGGGATTGG \\
\hline CALLOSE SYNTHASE 1 reverse & GAGCCGAACAAGAGTTGGAAGT \\
\hline GERMIN-LIKE 10 forward & TTACATGCAAGGCAGAGACG \\
\hline GERMIN-LIKE 10 reverse & AGGCCTGGGATTTTCTCAAC \\
\hline PICKLE-LIKE forward & TGGACTGTGAAATGCGCCCTAC \\
\hline PICKLE-LIKE reverse & ACTCTTTCTCAGGTACCCAAGTGC \\
\hline GH17_39 forward & AACCTGGGTTCAAGACAACG \\
\hline GH17_39 reverse & CGCATCTCCAGGGTGTACTT \\
\hline KIN2-LIKE forward & CTGACAATACCCAGAAG \\
\hline KIN2-LIKE reverse & CTGAGACATCACCTGTT \\
\hline
\end{tabular}

